\title{
SPECTROSCOPIC INVESTIGATION OF LIQUID HELIUM EXCITED BY A CORONA DISCHARGE: EVIDENCE FOR BUBBLES AND 'RED SATELLITES'
}

\author{
Z.-L. Li, N. Bonifaci ${ }^{1}$, F. Aitken, A. Denat, \\ G2E.lab, CNRS and Joseph Fourier University 25 rue des Martyrs, 38042 Grenoble, France \\ K. von Haeften, \\ University of Leicester, Department of Physics and Astronomy, University Road, Leicester, \\ United Kingdom
}

V.M. Atrazhev, Joint Institute for High Temperatures (JIHT) and Russian Academy of Sciences, Izhorskaya St. 13, 2125412, Moscow, Russia

V.A. Shakhatov

Topchiev Institute of Petrochemical Synthesis and Russian Academy of Sciences, Leninskii Prospect 29, 119991 Moscow, Russia)

\begin{abstract}
The establishment of corona discharges close to a point electrode under both negative and positive high voltage in normal liquid helium (LHe) at $4.2 \mathrm{~K}$ is reported. The experiments were carried out at constant temperature and pressures ranging from 0.1 $10 \mathrm{MPa}$. Visible luminescence emitted from the zone close to the tip revealed lines due to excited $\mathrm{He}$ atoms and molecules. The molecular luminescence showed hot band emissions with vibrational levels populated up to $\mathrm{v}=2$. Rotational temperatures of $800 \mathrm{~K}$ were estimated showing that the excitations do not thermalise. With increasing pressure the lines shifted to shorter wavelengths and became broader. The magnitude of the increase in width deviated from what is expected from the gas phase and from classical line broadening theory and rather showed similarities to the behavior of bubbles in LHe. The detailed analysis of the rotational line intensity distribution revealed the presence of an additional radiator at the long wavelength side of molecular bands that we tentatively assign to 'red satellite' emission. For corona discharges with positive tip polarities both atomic and molecular lines showed "red satellite" bands with much larger intensity than for negative polarity. The origin of the red satellite and the polarity dependence is unclear yet.
\end{abstract}

\section{INTRODUCTION}

Electrical discharge in dense dielectric liquids and high-pressure gases is a very complex process that involves electronic, thermal and hydrodynamic phenomena. The characteristics of the discharge

\footnotetext{
${ }^{1}$ nelly.bonifaci@grenoble.cnrs.fr
} 
are determined by the geometry of the electrode system, for instance, point-plane geometry is required to avoid spark breakdown within the inter-electrode space when a high electric voltage is applied. A specific advantage of the point-plane electrode geometry is that it makes the generation of discharges in very dense media, such as liquid helium (LHe), possible. The divergent spatial distribution of the electric field separates the charge formation region characterized by strong field strengths from the low field drift zone region where charge carriers drift without multiplication. The generation of the charges occurs very close to the tip electrode where the electric field is high enough for impact ionization of the fluid. The charge formation zone is usually much smaller than the inter-electrode gap. In the drift zone the electric current is determined by the carrier's mobility [1]. In high mobility liquids such as liquid Ar [2] the transient electric current of the corona discharge in the liquid reaches a magnitude of $0.16 \mathrm{~mA}$ [3]. To support such large currents intense ionization has to occur close to the tip electrode. The process is accompanied with the release of energy creating a gas-plasma ball in the small ionization region and the emission of luminescence. Spectroscopic analysis of the light emission from the ball allows us to estimate the temperature and the vapor density inside the non-equilibrium plasma formed by the corona discharge in liquid Ar [4]. Different to Ar the mobility of electrons and positive ions is very low in liquid $\mathrm{He}$ [5] and the electric current of the corona discharge in liquid $\mathrm{He}$ does not exceed $0.5 \mu \mathrm{A}[6]$. Such a low current gives hope that the plasma formation process is much less pronounced in liquid $\mathrm{He}$ than in liquid $\mathrm{Ar}$ and there is the possibility to produce localized excitations in the liquid phase which are known to produce intense luminescence in the visible and near infrared spectral range. This luminescence has been observed from superfluid ${ }^{4} \mathrm{He}$ bombarded with energetic electrons [7-9], from liquid helium excited by a corona discharge [10-13], from ${ }^{3} \mathrm{He}$ and ${ }^{4} \mathrm{He}$ clusters and droplets excited by monochromatic synchrotron radiation [14-16] and from liquid helium excited by femtosecond laser pulses $[17,18]$. We also note the recent photo excitation luminescence studies of LHe [19] as well as the pioneering work by Surko and Reif using vacuum ultraviolet (VUV) radiation [20].

In discharges the light emission process is believed to be initiated by electron avalanches in the strong field region leading to subsequent impact ionization of the fluid. The so-produced $\mathrm{He}^{+}$ions interact strongly with surrounding atoms leading to dimerisation $\mathrm{He}^{+}+\mathrm{He} \rightarrow \mathrm{He}_{2}^{+}$and dissociative recombination of the ions $\mathrm{He}_{2}^{+}+\mathrm{e}^{-} \rightarrow \mathrm{He}_{2}{ }^{*}$. Eventually, neutral excimers and, after further dissociation, excited $\mathrm{He}$ atoms are formed. These excited $\mathrm{He}$ atoms and $\mathrm{He}_{2}{ }^{*}$ excimers are the origin of the above mentioned luminescence. The visible luminescence stems from transition between high lying and lower-lying electronically excited states whereas the VUV radiation is due to transitions to the electronic singlet ground state. Radiative transitions between electronically excited states are usually very inefficient in condensed matter systems but in liquid $\mathrm{He}$ all excited $\mathrm{He}^{*}$ and $\mathrm{He}_{2}{ }^{*}$ reside in Rydberg states which gives rise to considerable structural rearrangement. This structural rearrangement is caused by the repulsive force between Rydberg electron orbitals and the surrounding ground state helium which as a consequence are pushed away within a short time [21,22] creating a void around the $\mathrm{He}^{*}$ and $\mathrm{He}_{2}{ }^{*}$. This void is often referred as a bubble and has typical radii between 10-14 $\AA$ depending on the electron's orbital radius [23]. These bubbles are very similar to the well known bubbles in liquid helium that form around localised electrons. Within the confinement of these bubbles the excitations are almost isolated. The perturbation by surrounding ground state helium atoms is low and the electronic life time of the excited atoms or excimers is similarly long as for free species in the vacuum. However, the remaining perturbation by the ground state helium atoms surrounding the bubble is still strong enough to cause broadening and wavelength shifts of the atomic and molecular lines and the magnitude of the width and shift was found to depend on applied pressure. To obtain a better understanding of these effects we have set up are corona discharge excitation scheme and initiated a systematic spectroscopic investigation of liquid and dense gaseous He. Our method has the potential for a relatively simple and versatile excitation source to investigate electronic excitations and luminescence in liquid helium as it allows changes of pressures and densities over a wide range.

In this paper we report spectra produced by corona discharges with point-plane electrode geometry in liquid helium at $4.2 \mathrm{~K}$ as a function of external hydrostatic pressure and the polarity of the electrodes. A rich spectrum, which contains atomic and excimer emissions from both singlet and triplet states has been observed. High resolution spectra recorded for low hydrostatic pressure in the LHe showed well 
resolved rotational structure of the molecular bands. The line intensity distribution of the rotationally resolved band was calculated for free molecules which revealed differences with respect to the excimer spectra in LHe. Surprisingly, these differences in line intensity depended on the polarity of the corona discharge. The hydrostatic pressure was also found to affect the line intensity distribution of the rotational spectrum of the confined $\mathrm{He}_{2}{ }^{*}$ as well as the total luminescence yield.

\section{EXPERIMENT}

Our experimental set up consisted of a cryostat that allowed us to control the temperature from room temperature to $4.2 \mathrm{~K}$ over a wide range of pressures up to $10 \mathrm{MPa}$. At the cold finger of the cryostat a $50 \Omega$ copper beryllium coaxial cell that could withstand pressures up to $10 \mathrm{MPa}$ was attached. The cell was evacuated using a turbo molecular pump to about $10^{-4} \mathrm{~Pa}$ and filled with ultra pure helium of grade N 60 (L'Air Liquide) which had an impurity concentration of less than $0.1 \mathrm{ppm}$ of oxygen. The gas was passed through a series traps filled with a mixture of molecular sieves (3-10 $\AA$ ) and charcoal, activated under vacuum typically at $350^{\circ} \mathrm{C}$ for 3 days, which were thereafter immersed in liquid $\mathrm{N}_{2}$.

The cell contained a point electrode that was made of an electrolytically-etched tungsten wire of 1 $\mathrm{mm}$ in diameter. The electrolytic etching resulted in tungsten tips with radii between $0.45 \mu \mathrm{m}$ and $2.5 \mu \mathrm{m}$ which were revealed by inspection with an electron microscope. The plane electrode was made of stainless steel and the plane electrode was always electrically connected to ground. The electrode spacing was $8 \mathrm{~mm}$. All metallic electrodes were supported by Macor insulators. Negative and positive high voltages from a stabilized DC power supply (Spellman RHSR/20PN60) were connected to the point electrode. Positive and negative corona discharges were realized by changing the polarity of the point electrode. A Tektronix TDS540 oscilloscope or a Keithley 610 C Ampere meter was connected to the plane electrode.

The light emitted from the corona region was transmitted through a sapphire window, collected using a lens and focused on the entrance slit of a spectrograph.

Spectra were recorded using an Acton Research Corporation spectrograph with $300 \mathrm{~mm}$ focal length. The spectrograph was equipped with 3 plane gratings of 150 grooves $/ \mathrm{mm}$ and two of 1200 grooves $/ \mathrm{mm}$, which were blazed at $750 \mathrm{~nm}$ and $300 \mathrm{~nm}$, respectively. A liquid nitrogen cooled 2DCCDTKB-UV/AR detector was located directly in the exit plane of the spectrograph. At the operating temperature of $153 \mathrm{~K}$ the dark current was lower than $1 \mathrm{e} / \mathrm{pixel} / \mathrm{h}$ resulting in a noise level that was only dominated by the read-out noise. The instrumental broadening was estimated by recording the spectral profiles of argon lines from a low pressure discharge lamp resulting in $\Delta \lambda_{\text {ins }}=0.12 \mathrm{~nm}$ for the 1200 grooves/mm grating.

\section{RESULTS AND DISCUSSION}

Spectra were recorded in the wavelength range from 400 to $1080 \mathrm{~nm}$. Some representative spectra recorded at $4.2 \mathrm{~K}$ and $0.1 \mathrm{MPa}$ are shown in figure 1. A large number of lines could be identified as due to transitions between excited atomic and molecular levels [24, 25]. Figure 2 shows a Grotrian diagram of the involved electronic states. The states are relatively dense and it can be seen that all upper levels of the atomic lines observed in the spectra have energies larger than $23 \mathrm{eV}$ and that the corresponding lower levels have energies close to $21 \mathrm{eV}$. All the molecular energy levels lie lower: the upper electronic states of the molecular bands are close to $21 \mathrm{eV}$ and lie near to the lower atomic states. Energy transfer is facilitated due to the high density of states and it is quite possible that the radiative relaxation of the high lying atomic states with $\mathrm{n}>3$ to the levels with principal quantum number $=2$ precedes the excimer emission and possibly also their formation by collisions.

Figure $1 \mathrm{~b}$ shows the spectral range from $560-630 \mathrm{~nm}$ which is crowded with a number of overlapping atomic and molecular band. The F-singlet and f-triplet molecular bands can be identified with emission from three different vibrational levels $(0--0),(1--1)$, and $(2--2)$ of the $f^{3} \Delta_{u}-b^{3} \Pi_{g}$ 
triplet. The presence of hot bands indicates vibrational temperatures of several $1000 \mathrm{~K}$ and thus relatively inefficient relaxation of the vibrational energy. Hot bands in the luminescence spectrum of electron bombarded liquid helium were reported by Dennis and co-workers [7]. They were also seen in the luminescence spectrum of photo excited He clusters and droplets [14]. The observation of hot bands gives some evidence for the presence of bubbles which impede the exchange of vibrational energy. The hot bands are shifted by up to $2 \mathrm{~nm}$ due to the different vibrational energies of the $\mathrm{f}^{3} \Delta_{\mathrm{u}}$ and $\mathrm{b}^{3} \Pi_{\mathrm{g}}$ states having vibrational constants $\omega_{\mathrm{e}}$ of $1706.8 \mathrm{~cm}^{-1}$ and $1769.1 \mathrm{~cm}^{-1}$, respectively [24]. The rotational constants $B_{e}$ of the $\mathrm{f}^{3} \Delta_{\mathrm{u}}$ and $\mathrm{b}^{3} \Pi_{\mathrm{g}}$ states are $7.23 \mathrm{~cm}^{-1}$ and $7.44 \mathrm{~cm}^{-1}$, respectively. At the resolution of our spectrometer rotational lines can only be partly resolved because transitions emerge from even and odd rotational levels eventually leading to many overlapping lines [26, 27]. The situation is different for the spectral range between 630 and $670 \mathrm{~nm}$ shown in figure 1c. This range is less crowded and shows only the $\mathrm{d}^{3} \Sigma_{\mathrm{u}}-\mathrm{b}^{3} \Pi_{\mathrm{g}}$ triplet and $\mathrm{D}^{1} \Sigma_{\mathrm{u}}-\mathrm{B}^{1} \Pi_{\mathrm{g}}$ singlet transitions with well resolved rotational structure. The rotational constants $\left(7.34 \mathrm{~cm}^{-1}\right.$ and $\left.7.45 \mathrm{~cm}^{-1}\right)$ of the involved states $\left(\mathrm{d}^{3} \Sigma^{+}{ }_{\mathrm{u}}\right.$ and $\mathrm{b}^{3} \Pi_{\mathrm{g}}$, resectively) are similar to those of the $\mathrm{f}^{3} \Delta_{\mathrm{u}}$ and $\mathrm{b}^{3} \Pi_{\mathrm{g}}$ states but due to the upper $\Sigma$-state only transitions from odd rotational $\mathrm{K}$ levels are allowed. This results in nicely resolved $\mathrm{P}$ and $\mathrm{R}$ lines that are spaced by $1 \mathrm{~nm}$.

Figure 1d shows the spectral range between 900 and $930 \mathrm{~nm}$. The $\mathrm{c}^{3} \Sigma_{\mathrm{g}}-\mathrm{a}^{3} \Sigma_{\mathrm{u}}$ and $\mathrm{C}^{1} \Sigma_{\mathrm{g}}-\mathrm{A}^{1} \Sigma_{\mathrm{u}}$ transition between the vibration states $(0--0)$ have been observed. The singlet $\mathrm{C}$ and $\mathrm{A}$ and the triplet $\mathrm{c}$ and a states have very similar potential curves. Therefore singlet and triplet rotational lines overlap quite strongly $[12,13]$.

We also investigated the effect of hydrostatic pressure. Whereas at low pressures atomic lines and molecular bands are well resolved a background continuum from 490 to $1200 \mathrm{~nm}$ appears when the pressure $\mathrm{p}$ exceeds 4.0 MPa. No lines and bands are observed for $p>5.0 \mathrm{MPa}$. Another effect of increasing pressure is significant line broadening and line shifting towards shorter wavelengths as shown at the example of the atomic $3 s^{3} \mathrm{~S}_{1}-2 \mathrm{p}^{3} \mathrm{P}$ triplet line at 706 in figure 3 . In helium gas line broadening effects due to increased pressure can be explained by classical theory. Both shift and broadening of lines are proportional to the gas density [28] and are adequately explained by repulsive interaction between an excited atom and surrounded atoms [29] using the "impact approximation" [31]. The line widths that are predicted by this theory are shown in Fig.4 together with the observed widths of the $706 \mathrm{~nm}$ atomic line recorded in LHe at $4.2 \mathrm{~K}$ as a function of the hydrostatic pressures. The density of the gas was determined for saturated vapor for the given pressures up to the critical pressure of $0.22 \mathrm{MPa}$ [30]. It can be seen that the measured increase in line width is much smaller than the prediction for saturated gas using classical line broadening theory. This distinction is an important hint that the lines are emitted from He atoms in bubbles within the liquid [23] and not from the gas phase. In other words in the tip region at $4.2 \mathrm{~K}$ the liquid does not vaporize due to the presence of a corona discharge. We note that the classical line broadening theory uses the assumption of homogeneously distributed gas. For comparison we have also reproduced the pressure dependence of the line width from figure 6 in the paper by Hickman, Steets and Lane [23]. Their data agrees nicely with our measured widths for small pressures giving further evidence for the presence of bubbles in LHe excited by corona discharges.

In the following we analyse the triplet $d-b$ transition. As can be seen in Fig. $1 c$ and as has been mentioned above this transition reveals much better resolved lines that all the other molecular spectral features which allows us to compare the measured spectrum with a simulation. The $\mathrm{d}-\mathrm{b}$ transition reveals a number of complications due to its triplet nature and therefore, we briefly review some of its spectroscopic characteristics. The $d-b$ transition is characterised by Hund's coupling case $b$ : the total angular momentum $\mathrm{J}$ is given by the vector sum of the electronic spin $\mathrm{S}$ and angular momentum of the molecule without spin $\mathrm{K}$ with $\mathrm{K}=\Lambda+\mathrm{N}$ where $\Lambda$ is the electronic angular momentum and $\mathrm{N}$ is the angular momentum of the nuclear rotational motion. The upper molecular $\mathrm{d}^{3} \Sigma^{+}$state has an electronic orbital moment $\Lambda=0$ and an electronic spin $S=1\left({ }^{3} \Sigma\right.$ state) with a multiplicity of $2 S+1=3$ (therefore the designation 'triplet'). The spin of the ${ }^{4} \mathrm{He}$ nucleus is zero therefore the rotational wave-functions of the $\mathrm{He}_{2}$-molecule must be "symmetric" [32]. The term $\mathrm{d}^{3} \Sigma^{+}$u is odd (subscript "u") and in order to produce a totally symmetric state the rotational state has to be odd as well. The symmetry of the rotational wave function of the $\Sigma^{+}$term is given by $(-1)^{\mathrm{K}}$. Therefore, quantum numbers of $\mathrm{K}$ of the allowed $\mathrm{d}^{3} \Sigma^{+} \mathrm{u}$ states have to be odd and they possess the values $\mathrm{K}=\Lambda+1, \Lambda+3, \Lambda+5, \ldots=1,3,5, \ldots$ Every $\mathrm{K}$ 
state then splits into three states with different total angular momentum numbers $\mathrm{J}=\mathrm{K}-1, \mathrm{~K}, \mathrm{~K}+1$, so $\mathrm{J}$ assumes the values $0,1,2$ (for $\mathrm{K}=1$ ) etc. Therefore, for a rotating molecule, triplet fine structures are expected even if $\Lambda=0$. The fine structure for the case $\Lambda=0, S=1\left({ }^{3} \Sigma\right.$ states) have been discussed theoretically by Kramers [33]. We further recall that the level with $\mathrm{J}=\mathrm{K}$ has maximal energy and the level of $\mathrm{J}=\mathrm{K}-1$ has minimal energy. The level of $\mathrm{J}=\mathrm{K}+1$ is between them. The spin-splitting of the ${ }^{3} \Sigma$-term of $\mathrm{He}_{2}$ molecule is very small $[26,34]$ and cannot be resolved in our measurements.

The lower molecular term $b^{3} \Pi_{g}$ of the transition has the electronic orbital momentum $\Lambda=1$ and is characterised by Hund's case (b) because the coupling energy of L and S is too small to resist the tendency of the rotation to cause $\mathrm{S}$ to become uncoupled from the electric axis and coupled to the rotational axis [35]. Therefore, $\mathrm{K}$ and $\mathrm{J}$ are 'good' quantum numbers and the smallest value of $\mathrm{K}$ is $\mathrm{K}=\Lambda=1$. Every state of ${ }^{3} \Pi$ term is two fold degenerated ( $\Lambda$-doubling) with alternating symmetry [32].

Different to the odd $\mathrm{d}^{3} \Sigma^{+}{ }_{\mathrm{u}}$ state the $\mathrm{b}^{3} \Pi_{\mathrm{g}}$ term is even (subscript ' $\mathrm{g}$ ') and as a consequence the rotational states with odd symmetry are absent because of the requirement that the total wave-function has to be symmetric. The quantum number $\mathrm{K}$ possesses the values $1,2,3,4, \ldots$ and every $\mathrm{K}$-state is split into three levels with different $\mathrm{J}$ quantum number: $\mathrm{J}=\mathrm{K}-1, \mathrm{~K}, \mathrm{~K}+1$. The total energy of levels with quantum numbers $\mathrm{J}$ and $\mathrm{K}$ can be expressed by the formula [32]

$$
E=T_{e}+\hbar \omega_{e}\left(v+\frac{1}{2}\right)+B_{e} K(K+1)+A_{e} \Lambda \frac{(J-S)(J+S+1)}{2 K(K+1)}
$$

where $T_{e}, \omega_{e}$ and $B_{e}$ are the spectroscopic constants that designate the electronic, vibronic and rotational energies, respectively. The last term in equation (1) relates to the triplet splitting with the splitting constant $A_{e}=-0.227 \mathrm{~cm}^{-1}$ [34] for the $b^{3} \Pi_{g}$ term. The energy spacing between multiple levels $\mathrm{J}=\mathrm{K}-1, \mathrm{~K}, \mathrm{~K}+1$ is very small and can be neglected for our simulation. The order of the levels with increasing energy is $\mathrm{K}=1: \mathrm{J}=0, \mathrm{~J}=1, \mathrm{~J}=2 ; \mathrm{K}=2: \mathrm{J}=1, \mathrm{~J}=2, \mathrm{~J}=3$; etc. as shown in Fig. 5 for low lying levels.

The allowed transitions are controlled by the selection rule $\Delta \mathrm{J}=\mathrm{J}$ ' - J" $=-1,0,+1$, where $\mathrm{J}$ ' relates to the upper level and $\mathrm{J}$ " to the lower level [36]. The selection rules are connected with specific branches of rotational lines in a molecular band: $\Delta \mathrm{J}=0$ means the Q-branch, $\Delta \mathrm{J}=+1$ means the Rbranch, and $\Delta \mathrm{J}=-1$ means the P-branch. Therefore, for transitions of the Q-branch J' equals J" (or $\mathrm{J}$ $=>\mathrm{J}$ ), for the R-branch J' equals J" +1 ( or $\mathrm{J}=>\mathrm{J}-1$ ), and for the P-branch J' equals $\mathrm{J}$ "-1 (or J => J+1). The selection rules for $\mathrm{K}$-quantum number are $\Delta \mathrm{K}=0, \Delta \mathrm{K}=+1, \Delta \mathrm{K}=-1$. The transition $\mathrm{J}$ ' $=0=>\mathrm{J}$ " $=$ 0 is forbidden [24]. The selection rules give rise for a number of lines: Lines with $\Delta \mathrm{K}=\Delta \mathrm{J}$ have high intensities and are called 'general branches' (shown in Fig.5 as solid arrows) belong to. There are nine general branches such as Q1, Q2, Q3, P1, P2, P3, R1, R2, R3. The subscript 1 (for example Q1) means, that transition occurs from the level with maximum value of $\mathrm{J}$ in the triplet. The subscript 3 (for example Q3) means, that transition occurs from the level with minimal value of $\mathrm{J}$ in the triplet. The upper term is $\mathrm{d}^{3} \Sigma^{+}{ }_{\mathrm{u}}$ and its states have odd K-numbers starting with 1 . The rotational constants of the $(0,0)$ vibrational band of the $\mathrm{d}^{3} \Sigma^{+}{ }_{\mathrm{u}}$ and $\mathrm{b}^{3} \Pi_{\mathrm{g}}$ terms are close $\left(7.34 \mathrm{~cm}^{-1}\right.$ and $7.45 \mathrm{~cm}^{-1}$ [24]) and therefore all rotational lines of the Q-branch are close as well and cannot be resolved. These overlapping lines form the intense central peak of the band. The lines of the R-branch have wavelengths shorter than the lines of the Q-branch. The R-lines start with $\mathrm{K}$ " $=3$ because $\mathrm{K}$ " $=\mathrm{K}$ ' 1 and the lowest allowed value of $\mathrm{K}^{\prime}$ and $\mathrm{K}$ ' is 1 . The $\mathrm{P}$ branch is on the long wavelength side and the P-lines start with $\mathrm{K}^{\prime}=1$. A level scheme with the allowed transitions is shown in figure 5 .

The general transitions are accompanied by so-called 'satellite transitions' with $\Delta \mathrm{K} \neq \Delta \mathrm{J}$. There are ten satellite branches for the transition $\mathrm{d}-\mathrm{b}$ of the $\mathrm{He}_{2}$ molecule [36] such as ${ }^{\mathrm{Q}} \mathrm{R}_{12},{ }^{\mathrm{Q}} \mathrm{P}_{32},{ }^{\mathrm{Q}} \mathrm{P}_{21},{ }^{\mathrm{Q}} \mathrm{R}_{23}$ for the Q branch, ${ }^{\mathrm{P}} \mathrm{Q}_{23},{ }^{\mathrm{P}} \mathrm{R}_{13},{ }^{\mathrm{P}} \mathrm{Q}_{12}$ for the $\mathrm{P}$ branch and ${ }^{\mathrm{R}} \mathrm{Q}_{32},{ }^{\mathrm{R}} \mathrm{P}_{31},{ }^{\mathrm{R}} \mathrm{Q}_{21}$ for the $\mathrm{R}$ branch. The superscript shows the value of $\Delta \mathrm{K}$, for example $\Delta \mathrm{K}=0$ for Q-satellites. The capital letter relates to $\Delta \mathrm{J}$, for example $\Delta \mathrm{J}=-1$ for an $\mathrm{P}$ satellite line. Q-, P-, and R-satellites have the same wavelengths as the corresponding lines of the general branches. Thus every rotational K-line is a superposition of three lines from the general branch and some lines from satellites.

The spectral energies of the P- and R- rotational lines relative to the central Q-peak are given by: 


$$
E_{K^{\prime}}-E_{K^{\prime \prime}}=\left\{B_{e}\left({ }^{3} \Sigma\right) \cdot K^{\prime}\left(K^{\prime}+1\right)-D_{v}\left({ }^{3} \Sigma\right)\left[K^{\prime}\left(K^{\prime}+1\right)\right]^{2}\right\}-\left\{B_{e}\left({ }^{3} \Pi\right) \cdot K^{\prime \prime}\left(K^{\prime \prime}+1\right)-D_{v}\left({ }^{3} \Pi\right)\left[K^{\prime \prime}\left(K^{\prime \prime}+1\right)\right]^{2}\right\}
$$

Here, $\quad B_{e}\left({ }^{3} \Sigma\right)=7.229 \mathrm{~cm}^{-1}, \quad B\left({ }^{3} \Pi\right)=7.3375 \mathrm{~cm}^{-1} \quad$ and $\quad D_{\mathrm{v}}\left({ }^{3} \Sigma\right)=\mathrm{D}_{\mathrm{v}}\left({ }^{3} \Pi\right)=5.25^{*} 10^{-4} \mathrm{~cm}^{-1}$ are the spectroscopic constants for $\mathrm{He}_{2}$ in the gas phase. The level with $\mathrm{K}^{\prime}=1$ is the lowest level allowed for the ${ }^{3} \Sigma$ upper state such like $K^{\prime \prime}=1$ for the lower ${ }^{3} \Pi$ state. The electronic energy between these levels is $\mathrm{E}_{0}=15623 \mathrm{~cm}^{-1}$ [26] and corresponds to the band head with the wavelength of $640.08 \mathrm{~nm}$. The spectral position of the Q-, P-, and R-lines calculated using Eq.(2) is shown in Fig. 6. The calculated wavelengths of the rotational lines are in a good agreement with the measured line frequencies reported in the literature [26]. Comparison of our measured spectrum with the position of the rotational lines of free molecule shows that the spectrum recorded for LHe at $4.2 \mathrm{~K}$ is shifted by 0.5 nm towards shorter wavelengths.

The rotational line intensity is proportional to

$$
I_{J^{\prime} J^{\prime \prime}} \propto S_{J^{\prime} J^{\prime \prime}} \cdot \operatorname{pop}\left(J^{\prime}\right)
$$

Here $S_{J^{\prime} J}$, is the Hönl-London factor [36], pop(J') is the population of the upper level J'. The HönlLondon factor depends on the total angular momentum $\mathrm{J}$ ' and $\mathrm{J}$ " of the transition and it is different for the general lines and the satellite lines which both contribute to the measured spectrum. The HönlLondon factors $S_{J^{\prime} J^{\prime}}$, of the b-d triplet rotation lines have been calculated in [37] and listed in [38] as a functions of the $\mathrm{J}$ "-number of the lower rotational level of transition. In our approximation the multiplet splitting of the rotational levels is not resolved and the (K', K') transition line intensity can be expressed as a function of the $\mathrm{K}^{\prime}$ quantum number of the upper triplet level. Thus, the line intensities for the Q-, P-, and R-branches can be expressed by the following simple formulae taking into account the contributions from general and satellite transitions

$$
I^{Q} K^{\prime} \propto\left(2 K^{\prime}+1\right) \cdot \operatorname{pop}\left(K^{\prime}\right), I^{P} K^{\prime} \propto\left(K^{\prime}+2\right) \cdot \operatorname{pop}\left(K^{\prime}\right), \quad I^{R} K^{\prime} \propto\left(K^{\prime}-1\right) \cdot \operatorname{pop}\left(K^{\prime}\right)
$$

Here $\mathrm{K}^{\prime}$ is the quantum number of the upper level of the rotational transition.

The analysis above allows us to estimate the population of the rotational levels. A simulated spectrum based on the distributions calculated by equation (4) is shown in figure 6 . The relative populations of rotational levels are presented in Fig.7 as a function of the quantum number K' in a semi-logarithmic plot. In this semi-logarithmic plot a Boltzmann distribution corresponds to a linear function. We find that a Boltzmann distribution is only exhibited for large K'. The 'rotational temperature' derived from this distribution is $780 \mathrm{~K}$ which is significantly higher than the temperature of $4.2 \mathrm{~K}$ of the liquid. Similar high rotational temperatures were observed for excimers ejected from photo excited ${ }^{3} \mathrm{He}$ and ${ }^{4} \mathrm{He}$ clusters and droplets $[14,15]$. Furthermore, the populations derived from the P-branch intensities are larger than those from R-branch lines intensities. This fact can formally be interpreted by the existence of an additional source of radiation. The magnitude of such a "red satellite" contribution can be obtained from the difference between measured and simulated spectra in the range of the P-branch lines. Contributions from higher vibrational transition such as $\left(v=1, v^{\prime}=1\right)$ can be excluded although its band head at $642 \mathrm{~nm}$ is close. The precise origin of the additional radiator is unclear yet and here we can only tentatively explain the red satellite bands as possibly due to a van der Waals bound molecular complex formed by the radiating atom or molecule and single helium atom.

Surprisingly, we observe a dependence on the corona discharge polarity. Spectra obtained with reversed polarity (positive tip voltage) show an increased intensity, Fig.8. Red satellites have been observed in the vicinity of both atomic and molecular lines. At present we do not have an explanation for the polarity dependence. Further calculations and experiments on the nature of the profiles of the red satellite lines and bands are in progress and will be reported together with a more detailed account of the results presented here. 


\section{CONCLUSION}

We have shown that, in liquid helium at $4.2 \mathrm{~K}$ localised atomic and molecular excited helium states can be created by using a corona discharge as excitation source. Spectra were recorded in a large range of hydrostatic pressures ranging from 0.1 to $4 \mathrm{MPa}$. The analysis of the observed shifts and widths shows a distinct behaviour from what is predicted by classical line broadening theory and provides evidence for the presence of emissions from species enclosed in bubbles. The analysis of the rotational line intensities of molecular bands reveals the existence of a new type of light emitter that contributes to the lower wavelengths of atomic and molecular bands. Surprisingly, the intensity of these 'red satellite' bands was found to depend on the corona discharge polarity. Further experiments and calculations are required to better understand the origin of this phenomenon.

\section{ACKNOWLEDGMENT}

Authors from Russia thank Russian Foundation of Basic Researches grant 08-08-00694 for support of their work.

\section{REFERENCES}

[1] R. S. Sigmond, J. Appl. Phys. 53, 892 (1982)

[2] J. A. Janke, L. Meyer, S. A. Rice, Phys. Rev. A 3, 734 (1971)

[3] N. Bonifaci, A. Denat, in Proceedings of the $12^{\text {th }}$ International Conference on Conduction and Breakdown in Dielectric Liquids, Roma, 1996, edited by C. Mazzetti (IEEE Catalog Number 96CH35981) p. 37

[4] N. Bonifaci, M. Nur, A. Denat, V. Atrazhev, C. Trassy, in Proceedings of 1999 IEEE $13^{\text {th }}$ International Conference on Dielectric Liquids, Nara, Japan, 1999, edited by K. Yoshino (IEEE Catalog Number 99CH36213) p. 441

[5] L. Meyer, H. T. Davis, Phys. Rev. 126, 1927 (1962)

[6] Z. Li, N. Bonifaci, A. Denat, V. Atrazhev, IEEE Trans. Dielectr. Electr. Insul. 13, 624 (2006)

[7] W. S. Dennis, E. Durbin, Jr, W. A. Fitzsimmons, O. Heybey, G.K. Walters, Phys. Rev. Lett. 23, 1083 (1969)

[8] F. J. Soley, W.A. Fitzsimmons, Phys. Rev. Lett. 32, 988 (1974)

[9] J. W. Keto, F.J. Soley, M. Stockton, W.A. Fitzsimmons, Phys. Rev. A 10, 872 (1974)

[10] P. H. Zimmermann, J. F. Reichert, Phys. Rev. B 15, 2630 (1977)

[11] V. A. Goncharov, V.I. Levitov, Izvestiya Academii Nauk, Energetika i Transport (in Russian) 12, 134 (1975)

[12] S. G. Kafanov, A. Ya. Parshin, I. A. Todoshenko, Sov. Phys. JETP 91, 1143 (2000)

[13] A. Ya. Parshin, I. A. Todoshenko, S. G. Kafanov, Physica B 91, 284 (2000)

[14] K. von Haeften, A. R. B. de Castro, M. Joppien, L. Moussavizdeh, R. von Pietrowski and T. Moller, Phys. Rev. Lett. 78, 4371 (1997)

[15] K. von Haeften, T. Laarmann, H. Wabnitz and T. Moller, Phys. Rev. Lett. 88, 233401 (2002)

[16] K. von Haeften, T. Laarmann, H. Wabnitz and T. Moller, J Phys. B: At. Mol. Opt. Phys. 38, S373 (2005)

[17] A. V. Benderskii, R. Zadoyan, N. Schwentner, V. A. Apkarian, J. Chem. Phys. 110, 1542 (1999)

[18] A. V. Benderskii, J. Eloranta, R. Zadoyan, V. A. Apkarian, J. Chem. Phys. 117, 1201 (2002)

[19] W. G. Rellergert, S. B. Cahn, A. Garvan, J. C. Hanson, W. H. Lippincott, J. A. Nikkel, D. N. McKinsey, Phys. Rev. Lett. 100, 025301-1 (2008)

[20] C. M. Surko, F. Reif, Phys. Rev. 175, 229 (1968)

[21] J. Eloranta, V. Apkarian, J. Chem. Phys. 117, 10139 (2002)

[22] M. Rosenblit, J. Jortner, Phys. Rev. Lett. 75, 4079 (1995) 
[23] A.P. Hickman, W. Steets, N. F. Lane, Phys. Rev. B 12, 3705 (1975)

[24] G. Herzberg, Molecular Spectra and Molecular Structure: I. Spectra of Diatomic Molecules, $2^{\text {nd }}$ edn. (Van Nostrand, Princeton, N. J., 1950)

[25] NIST ASD Output: Lines. http://physics.nist.gov/cgi-bin/ASD/lines1.pl

[26] M. L. Ginter, J. Molec. Spectrosc. 18, 321 (1965)

[27] J. Pfaff, M. H. Begemann, R. J. Saykally, Molec. Physics 52, 541 (1984)

[28] A. Corney, Atomic and Laser Spectroscopy, (Oxford 1977)

[29] Z LI, PhD Thesis, J. Fourier University Grenoble, (2008)

[30] NIST, http://webbook.nist.gov/cgi

[31] W.R. Hindmarsh, A.D. Petford, G. Smith, Proc. Roy. Soc., A297, 296 (1967)

[32] L. D. Landau and E. M. Lifshits, Quantum mechanics, $2^{\text {nd }}$ ed. (Pergamon, Oxford, 1987)

[33] G. Kramers, Zeits. Fur Physik 53, 422 (1929)

[34] C. Focsa, P. F. Bernath, R. Colin, J. Mol. Spectrosc. 191, 209 (1998)

[35] R. S. Mulliken, et al, Phys.Rev. 34, 1530 (1929)

[36] I. Kovacs, Rotational structure in the spectra of diatomic molecules, (London, Adam Higer Ltd., 1969)

[37] A. Budo, Z. Phys. 105, 579 (1937)

[38] A.Schadee, Communication from the Observatory at Utrecht, B.A.N., 17, no. 5, 311 (1964)

\section{Figure captions.}

Figure 1. Spectra recorded from $\mathrm{LHe}$ at $4.2 \mathrm{~K}$ and $0.1 \mathrm{MPa}$ at different wavelength ranges.

Figure 2. Grotrian of the atomic and $\mathrm{He}_{2}$ excimer levels. The arrows indicate the D-B singlet and d-b triplet transitions.

Figure 3. Profile of the $706 \mathrm{~nm}$ atomic line for different pressures at $4.2 \mathrm{~K}$.

Figure 4. Full-Width-at-Half-Maximum (FWHM) of the 706nm atomic line vs. pressure. Solid line: calculation using the 'bubble' model reproduced from figure 6 in [23]. Dashed line: calculation using the 'impact' approximation.

Figure 5. Schematic representation of energy levels and transitions between the $\mathrm{d}^{3} \Sigma^{+} \mathrm{u}$ and $\mathrm{b}^{3} \Pi_{\mathrm{g}}$. The general bands are shown as solid arrows. Three Q-branch satellite lines ${ }^{\mathrm{Q}} \mathrm{R}_{12}$ are shown as dashed arrows.

Figure 6. Position of the rotational lines for $(0-0)$ band of the $d^{3} \Sigma^{+}{ }_{u}=>b^{3} \Pi_{g}$ triplet emisson. The points represent the measured spectrum. The simulation of the spectrum is shown as a thin line. The additional 'red satellite' radiator is shown as a thick line.

Figure 7. Relative population of rotational levels of the upper term $\mathrm{d}^{3} \Sigma^{+}{ }_{\mathrm{u}}$.

Figure 8. Rotational spectra of $\mathrm{d}^{3} \Sigma_{\mathrm{u}}^{+}=>\mathrm{b}^{3} \Pi_{\mathrm{g}}$ transition for corona discharges with positive tip polarities in $\mathrm{LHe}$ at $\mathrm{T}=4.2 \mathrm{~K}, P=0.142 \mathrm{MPa}$. 
FIGURES.
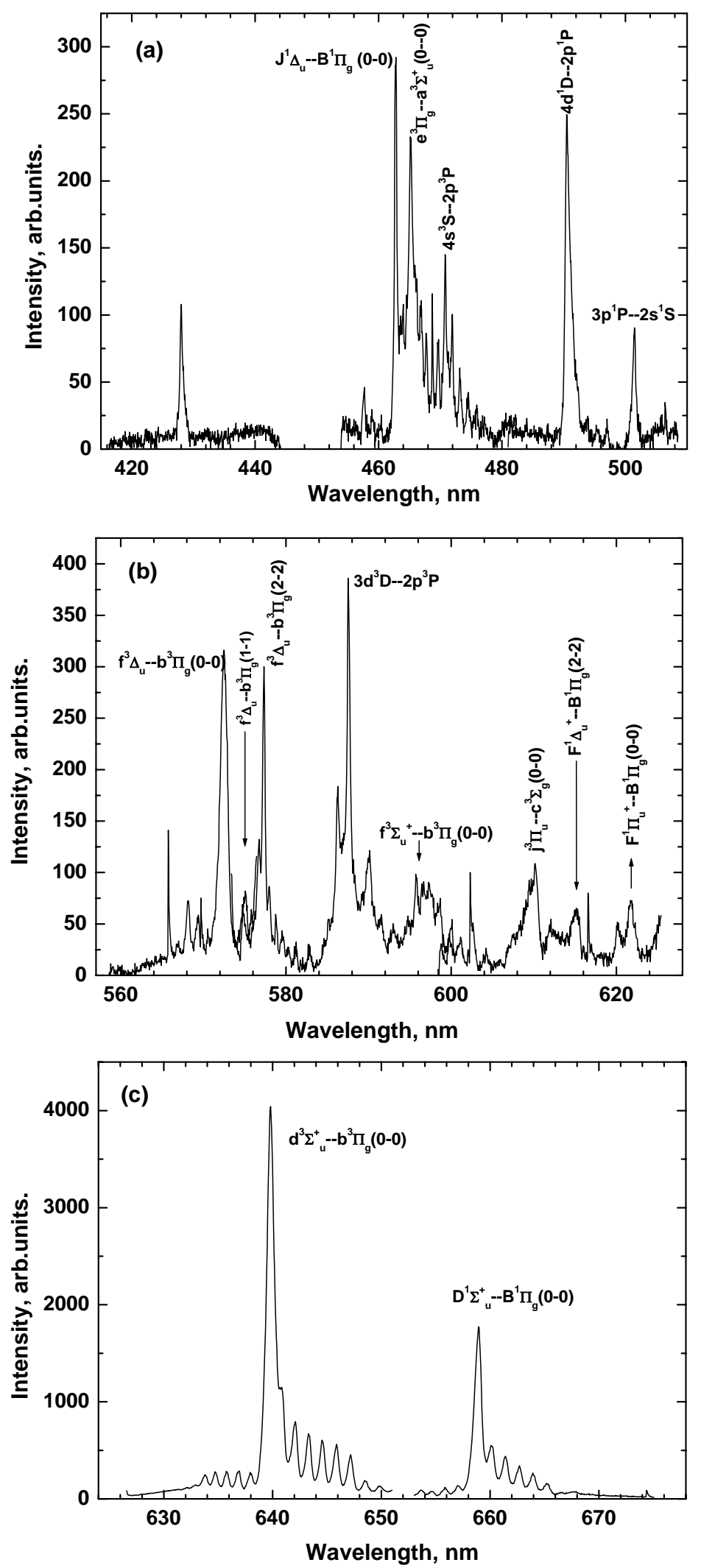


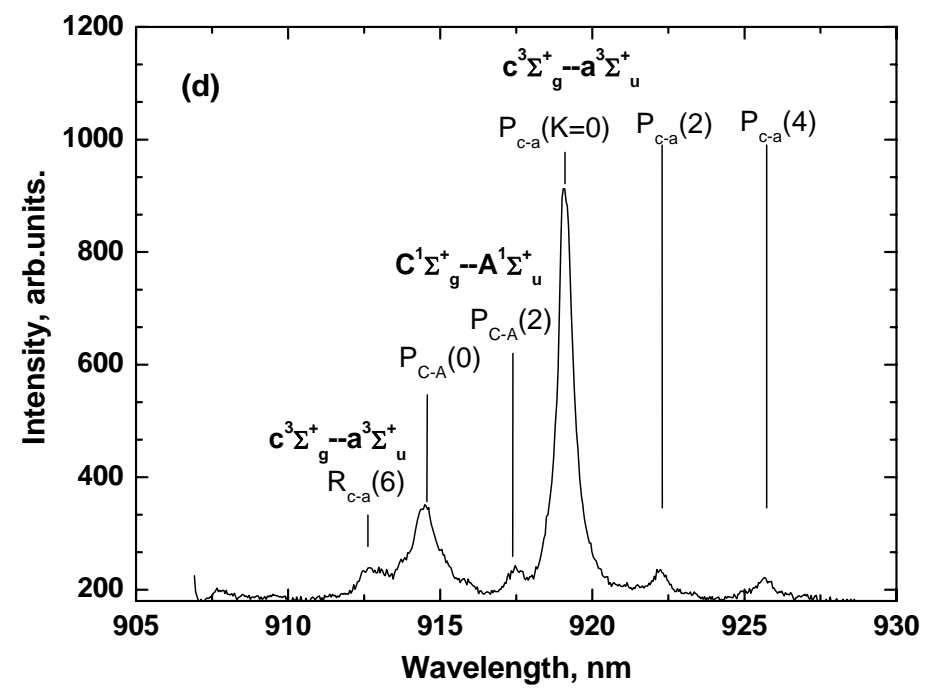

Figure 1. Spectra recorded from LHe at $4.2 \mathrm{~K}$ and $0.1 \mathrm{MPa}$ at different wavelength ranges.

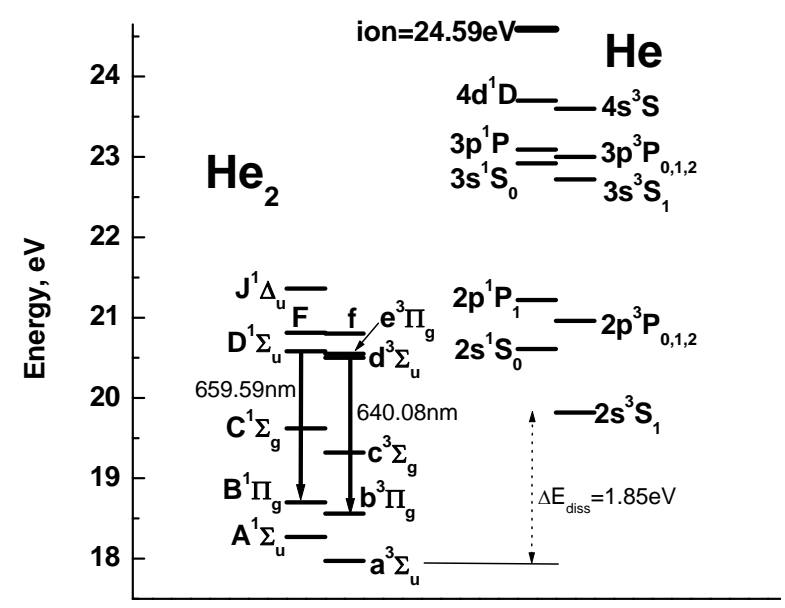

Figure 2. Grotrian of the atomic and $\mathrm{He}_{2}$ excimer levels. The arrows indicate the D-B singlet and d-b triplet transitions.

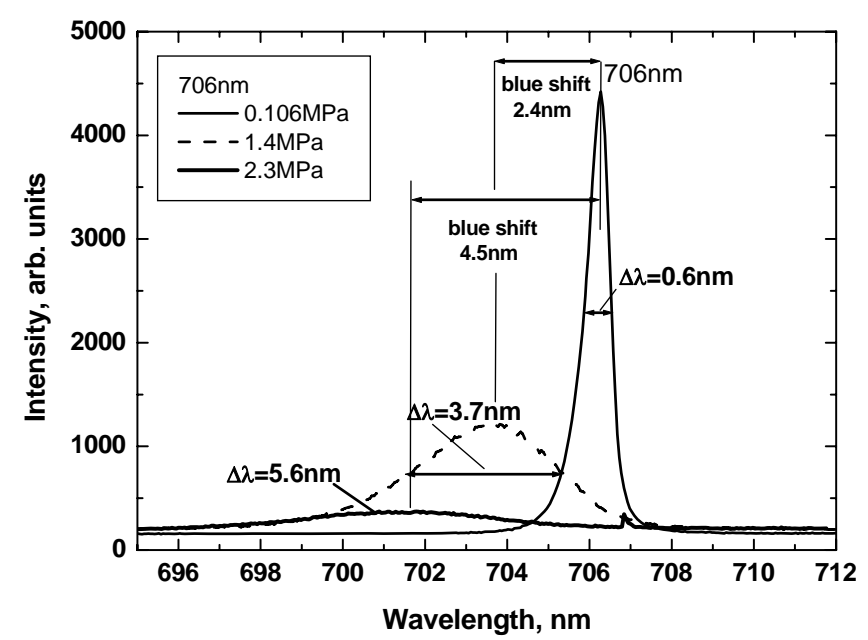

Figure 3. Profile of the $706 \mathrm{~nm}$ atomic line for different pressures at $4.2 \mathrm{~K}$. 


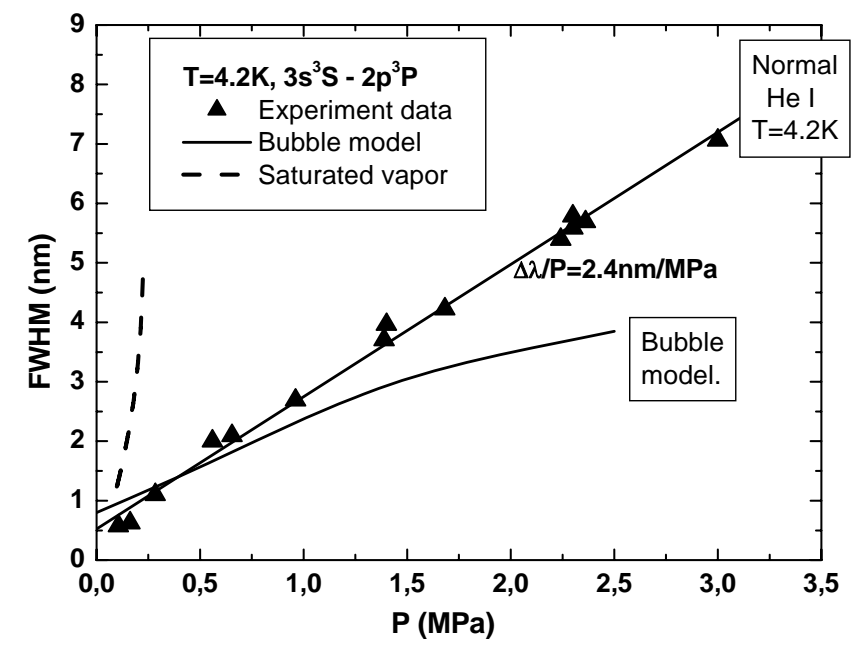

Figure 4. Full-Width-at-Half-Maximum (FWHM) of the 706nm atomic line vs. pressure. Solid line: calculation using the 'bubble' model reproduced from figure 6 in [23]. Dashed line: calculation using the 'impact' approximation.

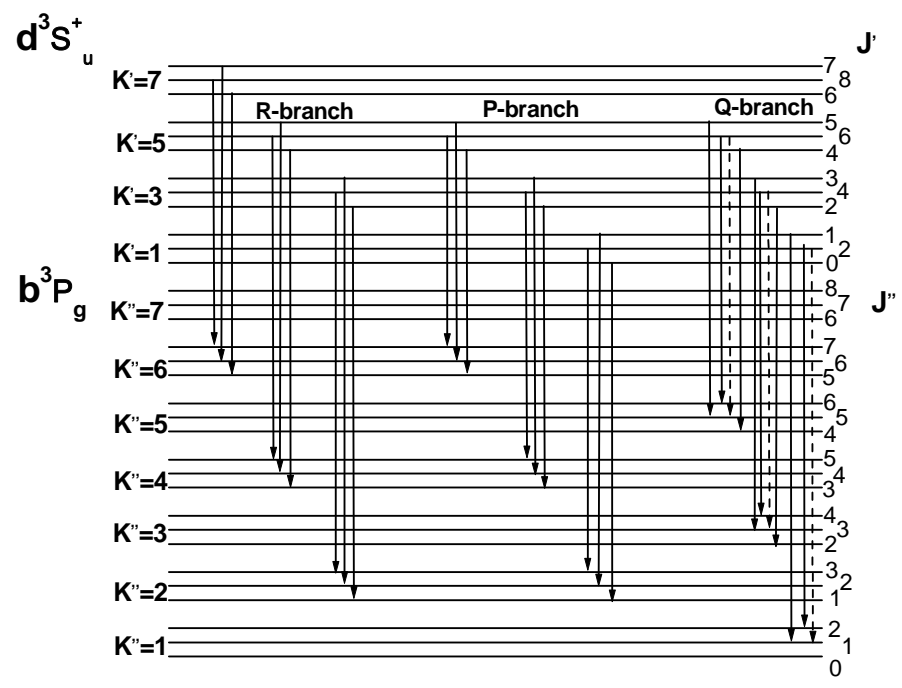

Figure 5. Schematic representation of energy levels and transitions between the $\mathrm{d}^{3} \Sigma^{+}{ }_{\mathrm{u}}$ and $\mathrm{b}^{3} \Pi_{\mathrm{g}}$. The general bands are shown as solid arrows. Three Q-branch satellite lines ${ }^{\mathrm{Q}} \mathrm{R}_{12}$ are shown as dashed arrows.

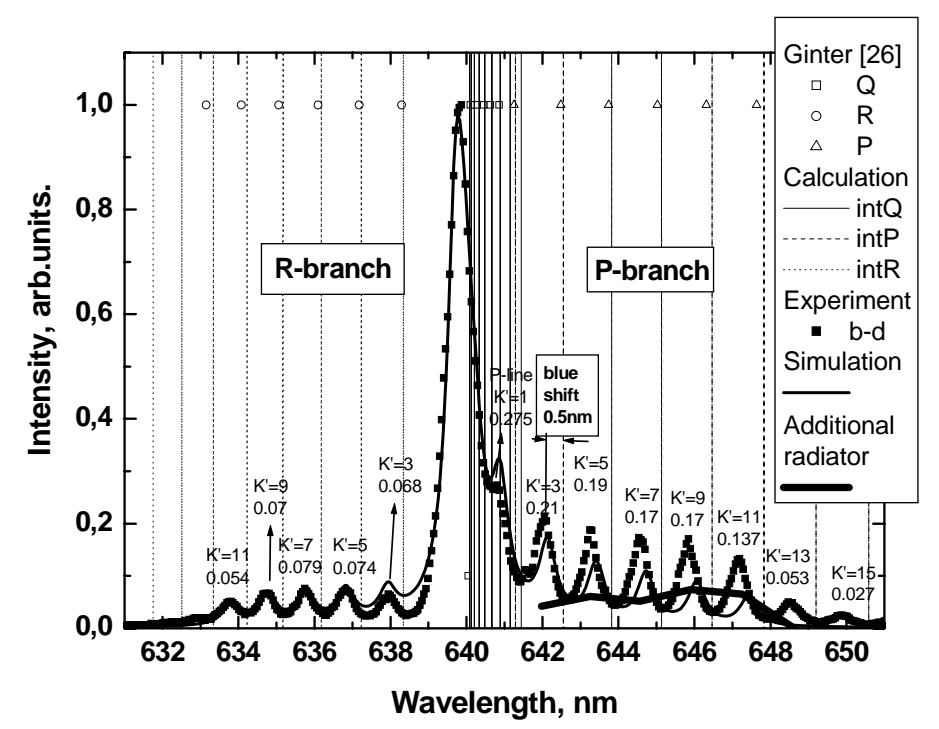


Figure 6. Position of the rotational lines for $(0-0)$ band of the $d^{3} \Sigma^{+}{ }_{u}=>b^{3} \Pi_{g}$ triplet emisson. The points represent the measured spectrum. The simulation of the spectrum is shown as a thin line. The additional 'red satellite' radiator is shown as a thick line.

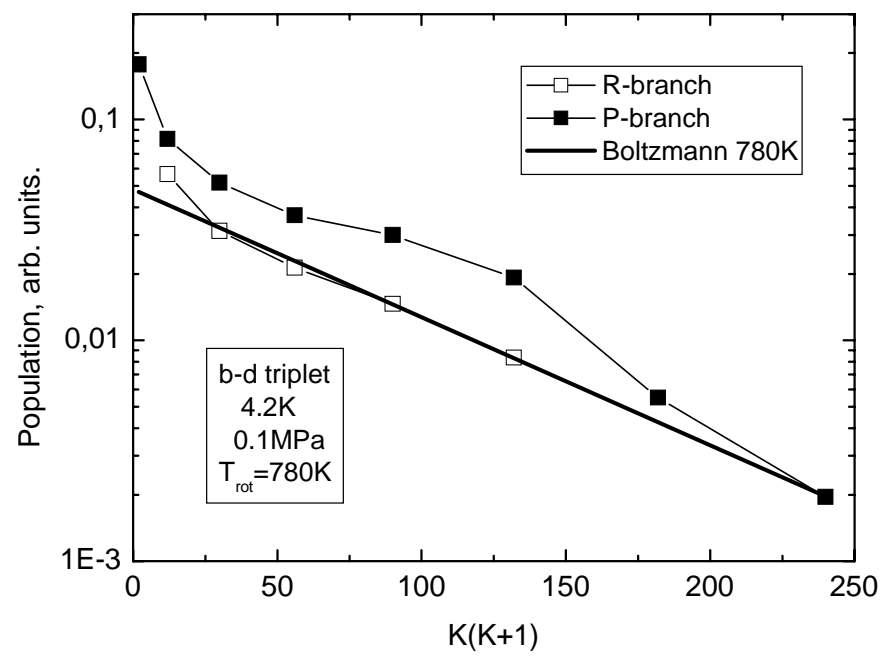

Figure 7. Relative population of rotational levels of the upper term $\mathrm{d}^{3} \Sigma^{+}{ }_{\mathrm{u}}$.

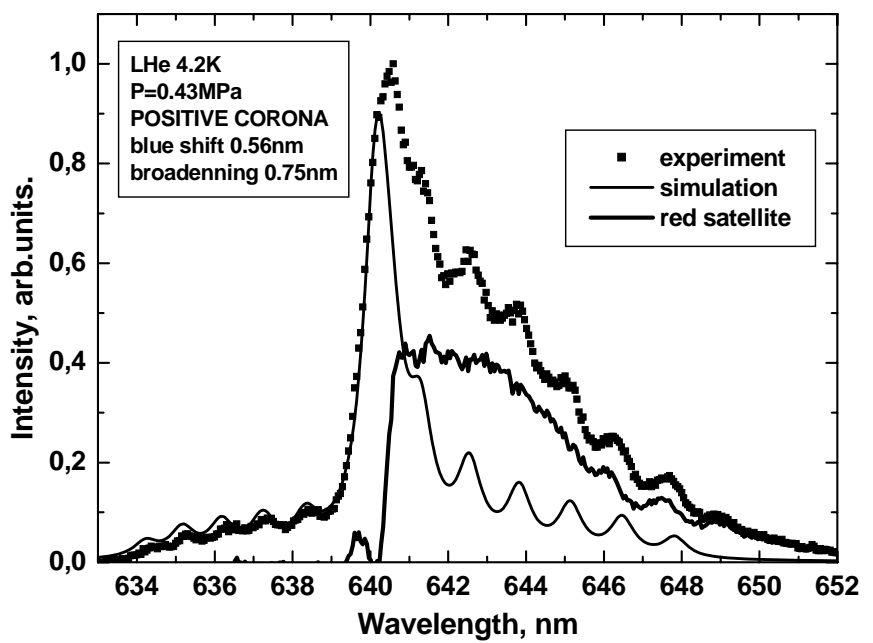

Figure 8. Rotational spectra of $\mathrm{d}^{3} \Sigma^{+}{ }_{\mathrm{u}}=>\mathrm{b}^{3} \Pi_{\mathrm{g}}$ transition for corona discharges with positive tip polarities in LHe at $\mathrm{T}=4.2 \mathrm{~K}, P=0.142 \mathrm{MPa}$. 